\title{
Development of an Index for Preventive Control Ranking and Selection for Voltage Stability Analysis
}

\author{
Khaled Alzaareer \\ Electrical Engineering Department \\ École de technologie supérieure \\ Montreal, Quebec, Canada \\ khaled.alzaareer.1@ems.etsmtl.ca
}

\section{Serge Lefebvre}

Power Systems and Mathematics

Research Institute of Hydro-Quebec

Montreal, Quebec, Canada

sergelefebvrefcpq@ireq.ca

\author{
Maarouf Saad \\ Electrical Engineering Department \\ École de technologie supérieure \\ Montreal, Quebec, Canada \\ Maarouf.saad@etsmtl.ca
}

Dalal Asber

Power Systems and Mathematics

Research Institute of Hydro-Quebec

Montreal, Quebec, Canada

Asber.Dalal@ireq.ca

\author{
Hasan Mehrjerdi \\ Electrical Engineering Department \\ Qatar University \\ Doha, Qatar \\ Hasan.Mehrjerdi@qu.edu.qa
}

\begin{abstract}
Preventive control selection for voltage stability concerns with determining the preventive controls that have to be activated, and finding a coordination between these preventive controls. This work develops an index, namely correction index $C I$, that can be used to rank the preventive controls for voltage stability analysis. This ranking helps the operators to select the most effective preventive controls to simultaneously mitigate the impacts of system contingencies on the critical buses. In other words, if such index is used for control selection, the useless (or less effective) controls can be ignored. This index is based on the degree of the effectiveness of the preventive controls to improve the voltage stability margin of power systems. The index can measure the efficiency of each preventive control not only to one bus (i.e. pilot bus), but to multiple critical buses of the system. The cost aspect is also involved in index calculation to distinguish between the cheap and the expensive controls. This means that the correction index helps the operators to select only the cheap and the higheffective controls. The proposed method is tested on the IEEE 39 bus network under a contingency scenario. The results show that the proposed index is accurate and valid for control ranking.
\end{abstract}

Keywords-Voltage stability; Preventive Control; Controls Ranking; Controls Coordination; Control selection, Smart grids.

\section{INTRODUCTION}

Voltage instability is one of the main problems in power networks. Many blackouts occurred around the worldwide due to voltage instability [1]. Thus, the development of assessment tools for voltage stability is necessary. This necessity is also raised with the development of distributed generation in power systems due to the variation in the power generation.

Once the contingency occurs on power systems, network operators have to take measures to mitigate contingency impacts on system voltage stability. If the distance to voltage instability is less than a specific threshold value, the contingency can be considered critical. If such critical contingency occurs on the power system, the network becomes insecure and control measures have to be applied to restore the system to a secure condition. It is worth mentioning that the voltage stability margin is usually determined by taking the difference between the highest loadability point and the present loading point.

Various approaches have been proposed to select the preventive or corrective controls for power systems [2]-[4]. One of these approaches is based on optimization problems for control selection for voltage stability analysis. The voltage stability margin is usually constrained through the optimization problem. The approach presented in [5] is an example of optimization-based methods.

However, a larger number of the preventive controls has to be used in the case of optimization-based methods. Another issue is that in case of dealing with multiple critical buses (instead of only pilot bus), the number of controls is further increased. This issue mainly occurs in the case of coordinated control schemes. Therefore, optimization approaches are not suitable for practical power systems with a large number of control variables.

To avoid the problems associated with optimization-based methods, some authors have developed techniques to choose the most efficient controls. These techniques are generally based on sensitivity analysis [6]-[12] of voltage stability margin (i.e. maximum loadability point) with respect to the preventive/correction controls. The controls can be then classified based on the sensitivity values.

The sensitivity analysis can usually rank the preventive controls for each individual bus of the system. However, they are not able to provide ranking and coordination in case of dealing with multiple critical buses. Considering only one bus of power system (i.e. pilot bus) for voltage stability analysis is not enough to ensure that the system is secure. This is due to the fact that the sensitivity analysis cannot provide the exact relation between the preventive controls and network buses. Actually, differences (i.e. errors) can be found between the obtained sensitivities and the actual dependences of the controls and network buses. This means that there is no guarantee that improving the voltage stability margin of pilot bus will make the system secure. There is possibly another critical bus in the system with a lower voltage stability margin. Moreover, with the development of fast response devices associated with distributed generators (i.e. power electronic devices), it is also necessary to consider a group of critical 
buses to reduce the errors in voltage stability assessment. The critical buses are usually defined as buses with lower voltage stability margins.

In general, there are three main tasks for efficient preventive control selection:

1. Ranking the preventive controls based on their effectiveness (and any other factors) in instability mitigation.

2. Determination of the preventive controls that have to be used for instability elimination according to a selection technique.

3. Control coordination between the selected preventive controls.

The target of this work concerns with task 1. Tasks 2 and 3 will be discussed in the next work.

To achieve task 1 and considering the aforementioned limitations, this work presents an index to rank the preventive controls to simultaneously mitigate the impacts of system contingencies on the critical buses. The index value represents the degree of the effectiveness of each preventive control with respect to critical buses. The effectiveness of the preventive controls mainly depends on the sensitivity of network buses with respect to preventive controls. The index is also formulated to measure the effectiveness to multiple critical buses, not only one bus. Besides, it includes control cost in the analysis. This index can be implemented for preventive control selection by sequentially choosing the controls, starting from the controls with higher ranks. By this strategy, cheap and high-effective preventive controls can be easily obtained for voltage instability mitigation.

The rest of this paper is organized as follows. Section II presents the sensitivity analysis approach used for the index. Section III presents the correction index formula. Section IV shows the simulation results and discussions while section $\mathrm{V}$ shows the conclusions.

\section{SENSITIVITY ANALYSIS}

With the evolution of monitoring devices, it is possible to design a model for online voltage stability prediction. The nodal currents of a multi-node power system can be expressed as:

$$
\left[\begin{array}{c}
I_{G} \\
-I_{L} \\
0
\end{array}\right]=\left[\begin{array}{lll}
Y_{G G} & Y_{G L} & Y_{G T} \\
Y_{L G} & Y_{L L} & Y_{L T} \\
Y_{T G} & Y_{T L} & Y_{T T}
\end{array}\right]\left[\begin{array}{c}
V_{G} \\
V_{L} \\
V_{T}
\end{array}\right]
$$

Where $I_{G}$ and $V_{G}$ are current and voltage vector of generator nodes, respectively. $I_{L}$ and $V_{L}$ are current and voltage vector of load nodes, respectively. $V_{T}$ is voltage vector of tie nodes. The terms $Y_{G G}, Y_{G L}, Y_{G T}, Y_{L G}, Y_{L L}, Y_{L T}, Y_{T G}$, $Y_{T L}, Y_{T T}$ are submatrices of the network admittance matrix $Y$.

The nodal currents presented in (1) can be extended to include the generator reactive power limits violation by including internal characteristics of generators. Thus, (1) becomes:

$$
\left[\begin{array}{c}
0 \\
-I_{L} \\
0 \\
I_{G}
\end{array}\right]=\left[\begin{array}{cccc}
Y_{G G}+Y_{n} & Y_{G L} & Y_{G T} & -Y_{n} \\
Y_{L G} & Y_{L L} & Y_{L T} & 0 \\
Y_{T G} & Y_{T L} & Y_{T T} & 0 \\
-Y_{n} & 0 & 0 & Y_{n}
\end{array}\right]\left[\begin{array}{c}
V_{G} \\
V_{L} \\
V_{T} \\
E_{G}
\end{array}\right]
$$

Where $E_{G}$ represents the internal voltage of the generator. $I_{G}$ is the generator current. $Y_{n}$ is a diagonal matrix. Each element of $Y_{n}$ represents the reciprocal of the impedance $Z_{G}$. $Z_{G}$ is the internal impedance of the generator.

According to [13], a voltage sensitivity margin $\Psi$ of bus i can be derived as:

$$
\Psi_{i}=\frac{\left|z_{L, i}\right|-\left|z_{e q, i}\right|}{\left|z_{L, i}\right|}
$$

Where $Z_{\text {eq }}$ and $Z_{L}$ are the equivalent and load impedances of system node "i", respectively, and can be calculated as presented in [13]. The values of $\Psi$ can be varied in the range $[0,1]$. The collapse point occurs when $\Psi=0$.

In this work, the same sensitivity approach proposed in [13] is also used for sensitivity analysis. The terms $\partial \Psi_{i} / \partial u_{k}$ is used to obtain sensitivity of voltage stability margin of bus $\mathrm{i}^{\text {th }}$ to the change in the preventive control " $\mathrm{k}$ ". Preventive controls can be capacitors and tap changers of the transformers, active and reactive power of generators or distributed generation units (i.e. power electronic devices).

For the change in a particular preventive control $\Delta u_{k}, \Delta \Psi_{i}$ can be expressed as:

$$
\Delta \Psi_{i}=\frac{\partial \Psi_{i}}{\partial u_{k}} \Delta u_{k}
$$

Where $\partial \Psi_{i} / \partial u_{k}$ is the sensitivity of $i^{\text {th }}$ voltage stability margin to $\mathrm{k}^{\text {th }}$ preventive control.

The normalized sensitivity between any preventive control "i" and bus $\mathrm{k}\left(\delta_{i k}\right)$ can be obtained as:

$$
\delta_{i k}=\frac{\partial \Psi_{i} / \partial u_{k}}{\max \left(\partial \Psi_{1} / \partial u_{k}, \partial \Psi_{2} / \partial u_{k}, \ldots, \partial \Psi_{n} / \partial u_{k}\right)}
$$

\section{FORMULATION OF THE CORRECTION INDEX}

In this section, the normalized sensitivities are utilized to develop an index (i.e. correction index) that ranks the system preventive controls for control selection. The correction index can measure the effectiveness of each preventive control for instability mitigation. The index depends on the voltage sensitivities of network buses with respect to the preventive controls.

The normalized sensitivity illustrated in (4) presents the effectiveness of each preventive control. For the control $u_{k}$, let $\boldsymbol{\delta}_{k}$ be the sensitivity vector of the multiple buses with respect to the preventive controls as:

$$
\boldsymbol{\delta}_{k}=\left[\boldsymbol{\delta}_{1 \mathrm{k}}, \boldsymbol{\delta}_{2 \mathrm{k}}, \ldots, \boldsymbol{\delta}_{\mathrm{ik}}, \ldots, \boldsymbol{\delta}_{\mathrm{nk}}\right]
$$

The cost of preventive controls can be involved in the index by modifying (5) as:

$$
\boldsymbol{\delta}_{x}^{\prime}=\frac{\boldsymbol{\delta}_{\mathrm{k}}}{C_{\mathrm{k}}}
$$

Where $C_{\mathrm{k}}$ is the unit cost of the $\mathrm{k}^{\text {th }}$ preventive control.

The corrective index CI of the preventive control can be found by taking the summation of $\boldsymbol{\delta}_{i k}^{\prime}$ as: 


$$
C I_{k}=\sum_{i=1}^{M} \boldsymbol{\delta}_{i k}^{\prime}
$$

Where $\mathrm{M}$ is the number of the multiple critical buses.

It is clear from (7) that the index takes into account the effectiveness, and cost aspects, and therefore it can measure the ability of the preventive controls to improve the voltage stability margins of multiple buses.

A flowchart of the correction index calculation is shown in Fig.1

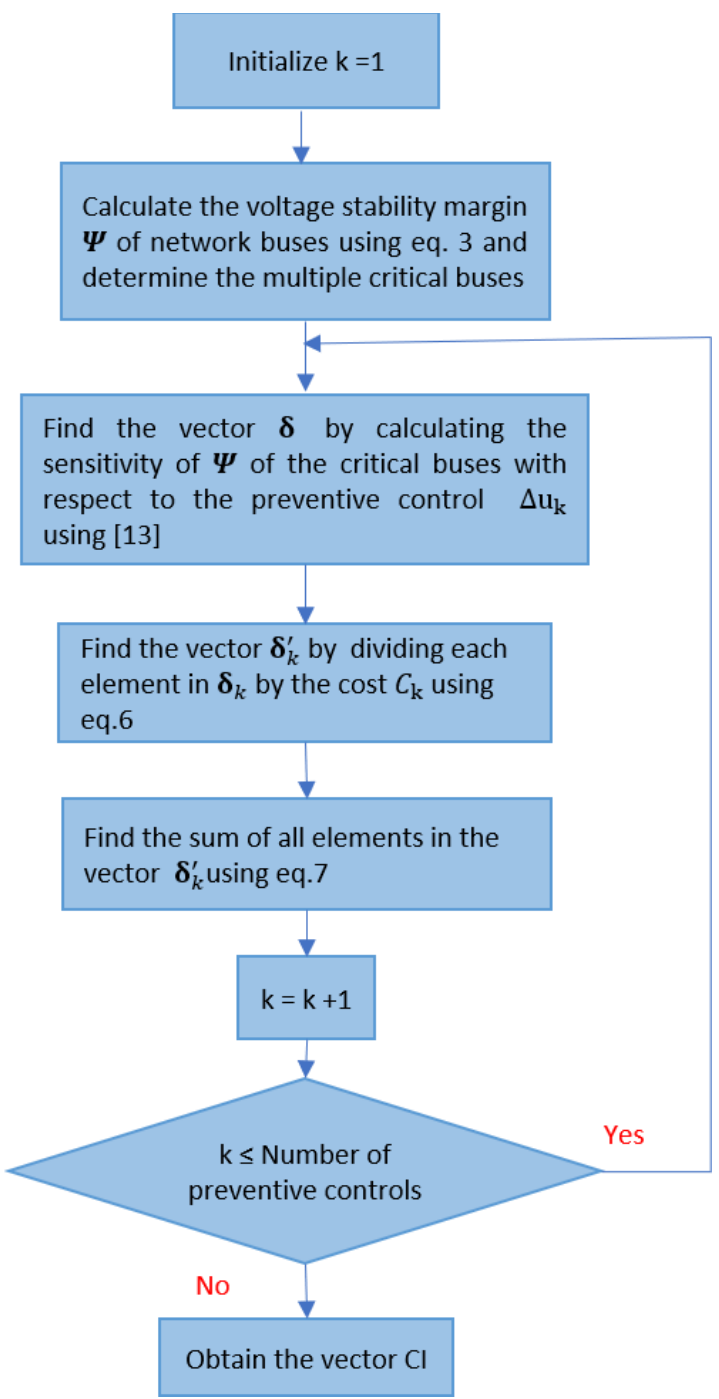

Fig.1: A flowchart of the correction index calculation

\section{Test System AND SimUlation RESUlts}

In this paper, IEEE 39-bus network is used to examine the validity of the correction index for voltage stability analysis of power system. The system topology is presented in Fig.2. This test system has 10 generators and 19 load buses. The slack is assumed to be bus 39. This means that the generator at bus 39 will not be included in the control ranking. It is also assumed that three capacitors (each with rating of 10MVar) are installed at three nodes $(5,15$, and 27$)$. The system is operated at a stressed scenario by multiplying the loads by 1.4. The network information can be found in [14]. MATLAB software is used to investigate the results.
In this study, the output power of the nine generators output and the three capacitors are considered as preventive controls. It is worth mentioning that the same procedure can be performed on other types of controls.

The preventive controls with their costs are presented in Table. I. This work assumes that the cost of using capacitors is smaller than the cost of using the output power of generators. The cost values represent the relative cost of each preventive control to provide ancillary services. To validate ability of the correction index to discriminate between the control variables, each type of preventive controls is also assigned with a different cost.

One contingency (line 3-18 outage) is considered in this work to check the validity of the proposed index in control ranking. At first, the index value for each preventive control is computed. According to the index values, a rank is then assigned for each control variable. The preventive control with a higher index value is assigned with a higher rank.

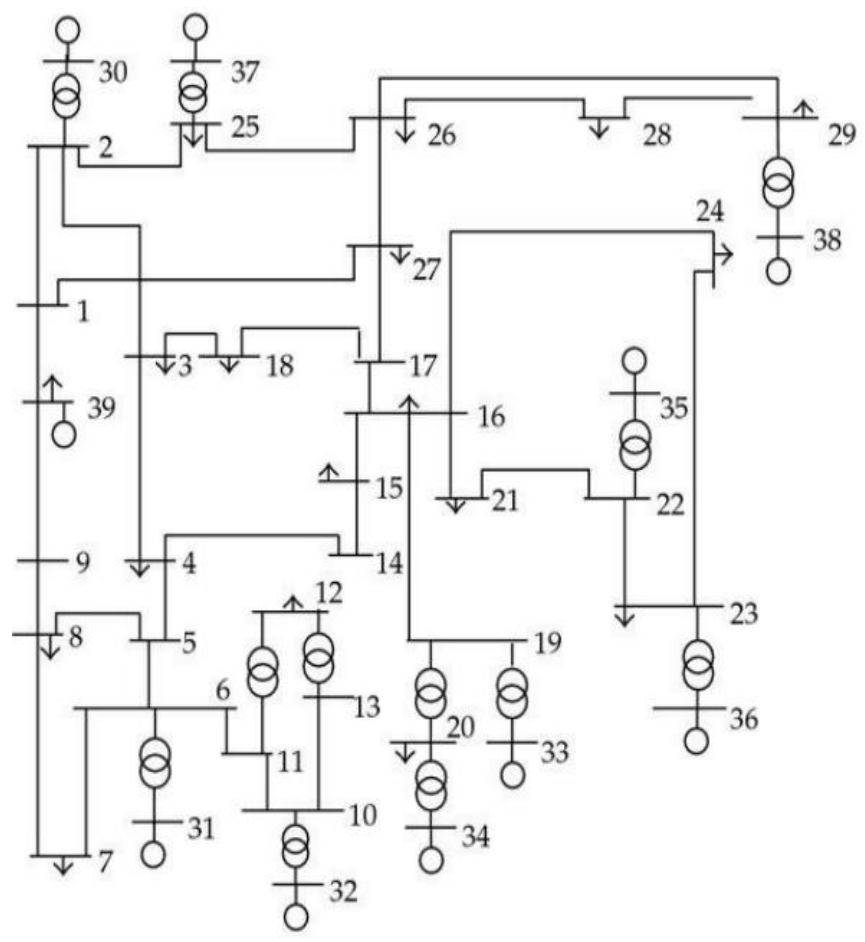

Fig. 2. The IEEE 39-bus system

TABLE I. Cost of The PREVEntive Controls

\begin{tabular}{|c|c|c|}
\hline $\begin{array}{c}\text { Preventive } \\
\text { Control }\end{array}$ & $\begin{array}{c}\text { Location } \\
\text { Bus no. }\end{array}$ & $\begin{array}{c}\text { Control } \\
\text { Cost }\end{array}$ \\
\hline$P_{g 1}$ & 30 & 100 \\
\hline$P_{g 2}$ & 31 & 110 \\
\hline$P_{g 3}$ & 32 & 100 \\
\hline$P_{g 4}$ & 33 & 90 \\
\hline$P_{g 5}$ & 34 & 90 \\
\hline$P_{g 6}$ & 35 & 100 \\
\hline$P_{g 7}$ & 36 & 100 \\
\hline$P_{g 8}$ & 37 & 85 \\
\hline$P_{g 9}$ & 38 & 95 \\
\hline$C_{1}$ & 5 & 20 \\
\hline$C_{2}$ & 15 & 20 \\
\hline$C_{3}$ & 27 & 20 \\
\hline
\end{tabular}


The ranking results using the correction index are also compared with the ranking obtained from continuation power flow (CPF) method [15]. CPF method is performed to find the maximum loadability points obtained by action of the preventive controls. These points can be easily obtained by inserting of each preventive control, at a time. The obtained maximum loadability points are then used to rank the preventive controls. The preventive control with higher maximum loadability (the preventive control has a higher effectiveness) is assigned with a higher rank.

\section{A. Calculation of Index Values}

The voltage stability margins on network buses are found by performing (3). In this work, only three buses (the buses having the lowest values of voltage stability margin) are selected to be considered as critical buses. From the analysis, it was found that the critical buses are 8,15 , and 16 . The CI values are calculated and presented in Fig.3. The control ranking is presented in Table II.

From Fig.3, it can be noticed that the shunt capacitors have higher ranks while the generators have low ranks. This means that the proposed index gives the priority for the capacitors for voltage instability mitigation. This is investigated by involving the cost in index calculation.

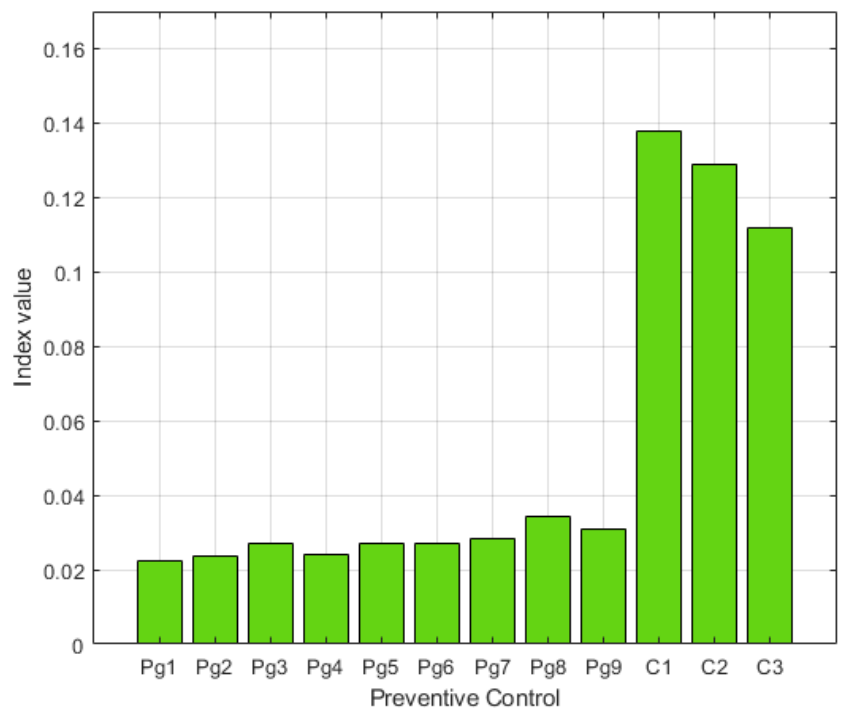

Fig.3. Values of the correction index of the preventive controls.

TABLE II. PREVEntive CONTROL RANKING InCluding The Cost

\begin{tabular}{|c|c|c|}
\hline $\begin{array}{c}\text { Preventive } \\
\text { Control }\end{array}$ & $\begin{array}{c}\text { CI } \\
\text { Index }\end{array}$ & $\begin{array}{c}\text { Control } \\
\text { Rank }\end{array}$ \\
\hline$P_{g 1}$ & 0.0222 & $(12)$ \\
\hline$P_{g 2}$ & 0.0237 & $(11)$ \\
\hline$P_{g 3}$ & 0.0269 & $(9)$ \\
\hline$P_{g 4}$ & 0.0241 & $(10)$ \\
\hline$P_{g 5}$ & 0.0270 & $(8)$ \\
\hline$P_{g 6}$ & 0.0272 & $(7)$ \\
\hline$P_{g 7}$ & 0.0285 & $(6)$ \\
\hline$P_{g 8}$ & 0.0343 & $(5)$ \\
\hline$P_{g 9}$ & 0.0310 & $(4)$ \\
\hline$C_{1}$ & 0.138 & $(1)$ \\
\hline$C_{2}$ & 0.129 & $(2)$ \\
\hline$C_{3}$ & 0.112 & $(3)$ \\
\hline
\end{tabular}

\section{B. Impact of Cost on the Index Value}

If the cost aspect was omitted from index calculation, the index calculation and the control ranking will be as shown in Table III. It is clear that shunt capacitors do not occupy the first ranks. This changing in the ranking demonstrates the necessary for involving the cost in index calculation.

TABLE III. PREVENTIVE CONTROL RANKING Without INCLUDING THE CosT

\begin{tabular}{|c|c|c|}
\hline $\begin{array}{c}\text { Preventive } \\
\text { Control }\end{array}$ & $\begin{array}{c}\text { CI } \\
\text { Index }\end{array}$ & $\begin{array}{c}\text { Control } \\
\text { Rank }\end{array}$ \\
\hline$P_{g 1}$ & 2.220 & $(11)$ \\
\hline$P_{g 2}$ & 2.607 & $(7)$ \\
\hline$P_{g 3}$ & 2.690 & $(6)$ \\
\hline$P_{g 4}$ & 2.169 & $(12)$ \\
\hline$P_{g 5}$ & 2.430 & $(9)$ \\
\hline$P_{g 6}$ & 2.720 & $(5)$ \\
\hline$P_{g 7}$ & 2.850 & $(3)$ \\
\hline$P_{g 8}$ & 2.915 & $(2)$ \\
\hline$P_{g 9}$ & 2.945 & $(1)$ \\
\hline$C_{1}$ & 2.760 & $(4)$ \\
\hline$C_{2}$ & 2.580 & $(8)$ \\
\hline$C_{3}$ & 2.240 & $(10)$ \\
\hline
\end{tabular}

\section{Comparison with CPF Results}

In this section, the ranking obtained using the correction index (without including the cost) is compared with the ranking from CPF. Fig.4 shows this comparison in a decreasing order. The ranks for both methods are expressed with numbers in parentheses. It is clear from the comparison that the correction index can correctly rank the preventive controls. The ranking using both methods are coincided. This demonstrates the accuracy of the correction index in selecting the high-effective preventive controls.

However, one important advantage of the proposed method is that the index calculation is faster than the traditional analysis (i.e. CPF), which makes it suitable for online applications.

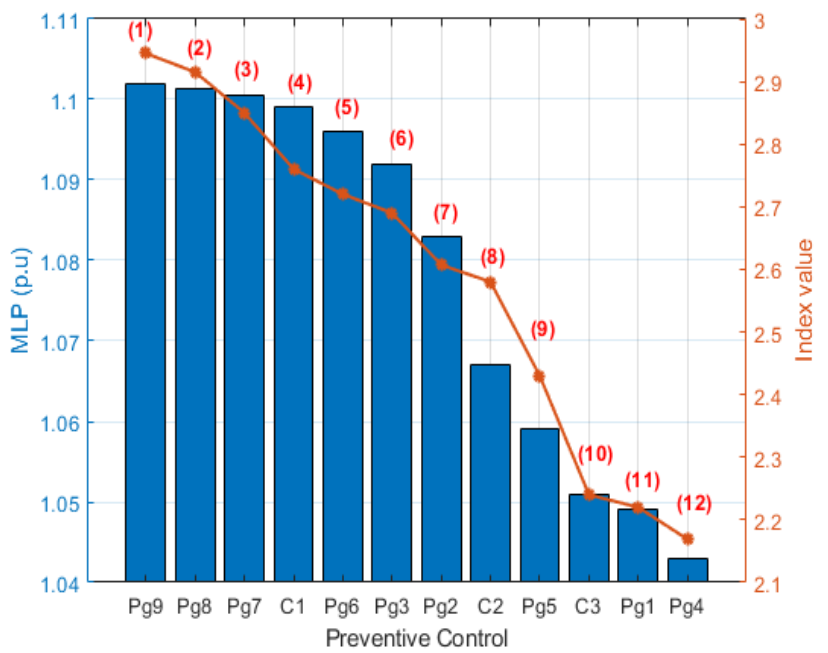

Fig.4. Comparison with the ranking obtained using CPF results. 
To the best of our knowledge, there are no other indices in the literature for ranking purpose. Thus, the proposed index is validated by comparing the obtained ranking with the one obtained via CPF.

\section{CONCLUSIONS}

This work presents an index for preventive control ranking for voltage stability analysis in power systems. The index takes into account the effectiveness and the cost of preventive controls. The index can measure the efficiency of each preventive control to multiple critical buses of the system.

Simulation was made on the IEEE 39 bus network. Different types of controls are included in the analysis. The ranking obtained using the index is compared with the ranking obtained from CPF results. The results show that the correction index is able to correctly rank the preventive controls based on their efficiency in case of the contingencies. The simulation results also show that including the cost aspect in the index calculation will affect the ranking results. As the cost of the preventive control increases, the index value decreases, and therefore the ranking may be changed. If such index is implemented for preventive control selection, the cheap and the high-effective controls will be easily obtained.

Our further work is to consider different types of preventive control. Development of new technique for control selection and based on the correction index is also part of our vision.

\section{ACKNOWLEDGMENT}

This work was supported by the National Priorities Research Program under Grant 11S-1125-170027 from the Qatar National Research Fund (a member of Qatar Foundation).

\section{REFERENCES}

[1] U. States and Canada, "Blackout in the United States and Canada: Causes and recommendations," Power System Outage Task Force, Apr. 2004

[Online].Available:http://energy.gov/sites/prod/files/oeprod/Documen tsandMedia/BlackoutFinal-Web.pdf,Tech.Rep.
[2] W.-M. Lin, W.-C. Hung, C.-H. Huang, and K.-H. Lu, "A preventive control for contingencies security,"in Proc. Int. Conf. Power Electron. Drive Syst., Nov. 2009, pp. 252-256.

[3] Chiang, H.D., Li, H., Tong, J. and Causgrove, P "On-line voltage stability monitoring of large power systems," in Proc. IEEE Power and Energy Soc. General Meeting,Jul.2011,pp.1-6.

[4] J. Zhao, Y. Wang, and P. Xu, "A comprehensive on-line voltage stability assessment method based on continuation power flow,"inProc. Int.Conf.Sustain.PowerGenerationandSupply,April2009,pp.1-5.

[5] X. Wang, G. Ejebe, J. Tong, and J. Waight, "Preventive/corrective control for voltage stability using direct interior point method," IEEE Trans.PowerSyst.,vol.13,no.3,pp.878-883,Aug.1998.

[6] J.Zhao,H.-D.Chiang, H.Li, and B.Zhang,"A novel preventive control approach for mitigating voltage collapse," in Proc. IEEE Power Eng. Soc. General Meeting, 2006,pp.2640-2645.

[7] S. Greene, I. Dobson, and F. Alvarado, "Sensitivity of the loading margintovoltagecollapsewithrespecttoarbitraryparameters,"IEEE Trans.Power Syst.,vol.12,no.1,pp.262-272,Feb.1997.

[8] B. Gao, G. K. Morison, and P. Kundur, "Toward the development of a systematic approach for voltage stability assessment of large-scale power systems," IEEE Trans. Power Syst., vol. 11, no. 3, pp. 13141324, Aug. 1996.

[9] M. Tang, Q. Zhu, K. Yang, H. Zhang, X. Li, Y. Huang, L. Chen, and W. Hu, "Simplified algorithm of voltage security correction based on sensitivity analysis method," in Proc. Power and Energy Eng. Conf., Mar. , pp. 1-4.

[10] B. Gao, G. K. Morison, and P. Kundur, "Voltage stability evaluation using modal analysis," IEEE Trans. Power Syst., vol. 7, no. 4, pp. 1529-1542, Nov. 1992

[11] T. Kumano, A. Yokoyama, and Y. Sekine, "Fast monitoring and optimal preventive control of voltage instability," Int. J. Electr. Power Energy Syst., vol. 1, pp. 117-125, Apr. 1994.

[12] Z. Feng, V. Ajjarapu, and D. J. Maratukulam, "A comprehensive approach for preventive and corrective control to mitigate voltage collapse,’IEEETrans.PowerSyst.,vol.3,no.2,pp.791-798,May2000.

[13] Alzaareer, Khaled, Maarouf Saad, Hasan Mehrjerdi, Claude Ziad ElBayeh, Dalal Asber, and Serge Lefebvre. "A new sensitivity approach for preventive control selection in real-time voltage stability assessment." International Journal of Electrical Power \& Energy Systems 122 (2020): 106212.

[14] IEEE 39-Bus Systems Description. Available at: https://icseg.iti.illinois.edu/power-cases/, accessed 1 May 2018.

[15] V. Ajjarapu, C. Christy "The continuation power flow: a tool for steady state voltage stability analysis”. IEEE Trans Power Syst, 7 (1) (1992), pp. $416-423$ 